\title{
A family of explicitly diagonalizable weighted Hankel matrices generalizing the Hilbert matrix
}

\author{
T. Kalvoda ${ }^{1}$, P. Štoviččk ${ }^{2}$ \\ ${ }^{1}$ Department of Applied Mathematics, Faculty of Information Technology, Czech \\ Technical University in Prague, Thákurova 9, 16000 Praha, Czech Republic \\ ${ }^{2}$ Department of Mathematics, Faculty of Nuclear Science, Czech Technical \\ University in Prague, Trojanova 13, 12000 Praha, Czech Republic
}

\begin{abstract}
A three-parameter family $B=B(a, b, c)$ of weighted Hankel matrices is introduced with the entries

$$
B_{j, k}=\frac{\Gamma(j+k+a)}{\Gamma(j+k+b+c)} \sqrt{\frac{\Gamma(j+b) \Gamma(j+c) \Gamma(k+b) \Gamma(k+c)}{\Gamma(j+a) j ! \Gamma(k+a) k !}},
$$

$j, k \in \mathbb{Z}_{+}$, supposing $a, b, c$ are positive and $a<b+c, b<a+c, c \leq a+b$. The famous Hilbert matrix is included as a particular case. The direct sum $B(a, b, c) \oplus B(a+1, b+1, c)$ is shown to commute with a discrete analog of the dilatation operator. It follows that there exists a three-parameter family of real symmetric Jacobi matrices, $T(a, b, c)$, commuting with $B(a, b, c)$. The orthogonal polynomials associated with $T(a, b, c)$ turn out to be the continuous dual Hahn polynomials. Consequently, a unitary mapping $U$ diagonalizing $T(a, b, c)$ can be constructed explicitly. At the same time, $U$ diagonalizes $B(a, b, c)$ and the spectrum of this matrix operator is shown to be purely absolutely continuous and filling the interval $[0, M(a, b, c)]$ where $M(a, b, c)$ is known explicitly. If the assumption $c \leq a+b$ is relaxed while the remaining inequalities on $a, b$, $c$ are all supposed to be valid, the spectrum contains also a finite discrete part lying above the threshold $M(a, b, c)$. Again, all eigenvalues and eigenvectors are described explicitly.
\end{abstract}

Keywords: weighted Hankel matrix; diagonalization; spectrum; Hilbert's matrix AMS Subject Classification: 47B35; 47B37; 47A10; 33C45 


\section{Introduction}

An integral operator $K$ on $L^{2}((0, \infty), \mathrm{d} x)$ whose integral kernel $\mathcal{K}(x, y)$ is real, symmetric and homogeneous of degree -1 on the first quadrant and such that

$$
\int_{0}^{\infty}|\mathcal{K}(t, 1)| t^{-1 / 2} \mathrm{~d} t<\infty
$$

is bounded and explicitly diagonalizable by the Mellin integral transform. In more detail, $K$ is unitarily equivalent to the multiplication operator by the function

$$
g(\xi)=\int_{0}^{\infty} \mathcal{K}(t, 1) t^{-1 / 2-i \xi} \mathrm{d} t=\int_{\mathbb{R}} e^{-i \xi x} \mathcal{K}\left(e^{x / 2}, e^{-x / 2}\right) \mathrm{d} x
$$

acting on $L^{2}(\mathbb{R}, \mathrm{d} \xi)$. This feature can readily be understood if the symmetry properties of such an integral operator are examined. $K$ commutes with the one-parameter unitary group of dilatation transformations on the positive half-line generated by the skew-symmetric differential operator $D=x \mathrm{~d} / \mathrm{d} x+1 / 2$. The kernel of the Mellin integral transform is in fact nothing but a family of generalized eigenfunctions of $D$.

All what has been said above is applicable to the integral kernel

$$
\mathcal{K}_{\ell}(x, y)=\frac{(x y)^{\ell / 2}}{(x+y)^{\ell+1}}
$$

depending on a real parameter $\ell$ provided $\ell>-1$. The corresponding integral operator $K_{\ell}$ is unitarily equivalent to the multiplication operator by the function

$$
g(\xi)=\int_{0}^{\infty} t^{(\ell-1) / 2+i \xi}(t+1)^{-\ell-1} \mathrm{~d} t=\frac{1}{\Gamma(\ell+1)}\left|\Gamma\left(\frac{1}{2}(\ell+1)+i \xi\right)\right|^{2} .
$$

In no way it is straightforward to find an authentic discrete analog of the integral kernel (11). Of course, given a homogenous kernel of degree -1 one can always restrict the kernel to the discrete set $\left(\theta+\mathbb{Z}_{+}\right) \times\left(\theta+\mathbb{Z}_{+}\right)$, for some $\theta>0$ and with $\mathbb{Z}_{+}$standing for nonnegative integers, obtaining this way a semi-infinite matrix. For example, using the kernel (1), with $\ell=0$, we get the (generalized) Hilbert matrix. Matrices of this type have been explored, for instance, in [7]. But as emphasized in the introduction of the cited paper, it appears that there may be inconveniences in applying to matrices some methods originally invented for integral operators. In particular, let us note that no obvious discrete analog of the Mellin integral transform yielding a diagonalization of such matrix operators is at our disposal. Nevertheless, comparison of a matrix operator obtained by discretizing an integral kernel with the integral operator in question can provide, under certain additional assumptions, quite a useful information including the precise value of the norm, see Chp. 1 in [14].

The present paper actually aims to describe matrix operators whose character and properties remarkably closely resemble those of the integral operators $K_{\ell}$. Notably, despite of nontrivial form of such matrices all spectral properties are known in full detail. Our approach is based on a construction of a three-parameter family of matrices 
$B=B(a, b, c)$ with the desired structure of weighted Hankel matrices and enjoying an appropriate symmetry. By the symmetry we mean a discrete analog $D$ of the generator of dilatation transformations which is commuting with $B$. $D$ can be regarded as a first order difference operator. Then $D^{2}$ commutes with $B$ as well and, since this is a second order difference operator, as such can be related to a Jacobi (tridiagonal) matrix. Owing to the discrete symmetry $D$, an explicit diagonalization of $B$ is possible. To this end, one has to rely on the theory of orthogonal polynomials in place of the Mellin transform. This is only roughly the main idea which is made precise and fully developed in the remainder of the paper.

The famous generalized Hilbert matrix is included in the family $B(a, b, c)$ as a one-parameter subfamily. The constructed diagonalization procedure if applied to Hilbert's matrix reproduces, in an alternative way, a complete solution to the spectral problem due to Rosenblum [12]. Considering only the original Hilbert matrix, i.e. not treating the whole one-parameter family, it has been observed earlier by Otte that a commuting Jacobi matrix can be used for a diagonalization. This result exists in the form of a conference presentation which is currently available from author's web page [10. But the first one who has discovered that the generalized Hilbert matrix has a tridiagonal matrix in its commutant seems to be Grünbaum in [6]. As noted in the cited paper, the class of Hankel matrices with this property is very limited. Saying this, the author was guided by his forgoing studies of a similar problem for the class of Toeplitz matrices [5].

\section{A family of weighted Hankel matrices with a dis- crete symmetry}

Consider the three-parameter family of semi-infinite symmetric matrices $B=B(a, b, c)$,

$$
B_{j, k}=\frac{\Gamma(j+k+a)}{\Gamma(j+k+b+c)} \sqrt{\frac{\Gamma(j+b) \Gamma(j+c) \Gamma(k+b) \Gamma(k+c)}{\Gamma(j+a) j ! \Gamma(k+a) k !}}, j, k \in \mathbb{Z}_{+} .
$$

The parameters $a, b$ and $c$ should be restricted to a range for which the matrices $B(a, b, c)$ are real and hence Hermitian. Throughout the paper we shall assume that the parameters $a, b, c$ are all positive. The entries of $B$ have the structure $B_{j, k}=$ $w(j) w(k) h(j+k)$ and therefore the matrices may be classified as weighted Hankel matrices, see Chp. $6 \S 8$ in [11].

Note that, by the Stirling formula, the leading asymptotic term of the matrix entry $B_{j, k}$ for both $j$ and $k$ large is

$$
B_{j, k} \sim \frac{(j k)^{(b+c-a-1) / 2}}{(j+k)^{b+c-a}} .
$$

This may be compared with the integral kernel (1), with $\ell=b+c-a-1$, suggesting that we should suppose

$$
b+c-a>0
$$


The matrix entries $B_{j, k}$ take particularly simple form if we put $a=\theta, b=\theta+\ell$, $c=1$, with $\ell \in \mathbb{Z}_{+}$. Then

$$
B_{j, k}=\frac{\sqrt{(j+\theta)_{\ell}(k+\theta)_{\ell}}}{(j+k+\theta)_{\ell+1}}
$$

where $(a)_{\ell}$ is the usual Pochhammer symbol. In particular, for $\ell=0$ we obtain the generalized Hilbert matrix, $B_{j, k}=1 /(j+k+\theta)$; for a short account on its history see Chp. IX in 8.

Moreover, letting $\ell=1$ we get a modification of the so called Bergman-Hilbert matrix,

$$
B_{j, k}=\frac{\sqrt{(j+\theta)(k+\theta)}}{(j+k+\theta)(j+k+\theta+1)},
$$

studied in [4, 3].

Put $A=B(a, b, c) \oplus B(a+1, b+1, c) . A$ can be identified with the semi-infinite matrix with the entries

$$
A_{2 j, 2 k}=B(a, b, c)_{j, k}, A_{2 j+1,2 k+1}=B(a+1, b+1, c)_{j, k}, j, k=0,1,2, \ldots,
$$

and $A_{j, k}=0$ if $j, k$ are of different parity. This identification corresponds to the direct sum

$$
\ell^{2}\left(\mathbb{Z}_{+}\right)=\ell^{2}\left(2 \mathbb{Z}_{+}\right) \oplus \ell^{2}\left(2 \mathbb{Z}_{+}+1\right) .
$$

$\left(\ell^{2}\left(2 \mathbb{Z}_{+}\right)\right.$is spanned by $\left\{e_{2 k} ; k \in \mathbb{Z}_{+}\right\}, \ell^{2}\left(2 \mathbb{Z}_{+}+1\right)$ is spanned by $\left\{e_{2 k+1} ; k \in \mathbb{Z}_{+}\right\}$ where $\left\{e_{k} ; k \in \mathbb{Z}_{+}\right\}$is the standard basis in $\left.\ell^{2}\left(\mathbb{Z}_{+}\right)\right)$. Furthermore, let us introduce another three-parameter family of matrices, $D=D(a, b, c)$, such that

$$
D_{j, j+1}=-D_{j+1, j}=d(j), D_{j, k}=0 \text { otherwise }
$$

where

$$
d(2 j)=\sqrt{(j+a)(j+b)}, d(2 j+1)=\sqrt{(j+1)(j+c)}, j, k=0,1,2, \ldots
$$

$D$ can be regarded as a discrete analog of the dilatation operator.

With respect to the decomposition (5), the matrices $A$ and $D$ can be written in the blockwise form

$$
A=\left(\begin{array}{cc}
B(a, b, c) & 0 \\
0 & B(a+1, b+1, c)
\end{array}\right), D=\left(\begin{array}{cc}
0 & C \\
-C^{T} & 0
\end{array}\right),
$$

where $C$ is another semi-infinite matrix with the entries

$$
C_{j, j}=d(2 j), C_{j+1, j}=-d(2 j+1), C_{j, k}=0 \text { otherwise. }
$$

Lemma 1. $A$ and $D$ commute. 
Proof. The proof can be carried out relying just on a straightforward evaluation of the matrix entries of the commutator. One has (with $d(-1):=0$ )

$$
(A D-D A)_{j, k}=d(k-1) A_{j, k-1}-d(k) A_{j, k+1}+d(j-1) A_{j-1, k}-d(j) A_{j+1, k} .
$$

Obviously, $(A D-D A)_{j, k}$ vanishes whenever $j$ and $k$ are of the same parity. Without loss of generality it suffices to consider the situation when $j$ is even and $k$ is odd. Let us suppose $j, k \in \mathbb{Z}_{+}$and evaluate the expression

$$
\begin{aligned}
(A D-D A)_{2 j, 2 k+1}= & d(2 k) B(a, b, c)_{j, k}-d(2 k+1) B(a, b, c)_{j, k+1} \\
& +d(2 j-1) B(a+1, b+1, c)_{j-1, k}-d(2 j) B(a+1, b+1, c)_{j, k} .
\end{aligned}
$$

A direct computation actually shows that the expression equals zero.

One may prefer, however, to proceed another way revealing the algebraic structure behind the identity. In view of (6),$A D-D A=0$ is equivalent to

$$
B(a, b, c) C=C B(a+1, b+1, c),
$$

i.e. $C$ intertwines the operators $B(a, b, c)$ and $B(a+1, b+1, c)$. In order to take into account the structure of $B=B(a, b, c)$ let us write $B=W H W$ where $W$ is the diagonal matrix with the diagonal entries

$$
W_{j, j}=\sqrt{\frac{\Gamma(j+b) \Gamma(j+c)}{\Gamma(j+a) j !}},
$$

and $H$ is the Hankel matrix, $H_{j, k}=h(j+k)$, where

$$
h(z)=\frac{\Gamma(z+a)}{\Gamma(z+b+c)} .
$$

Similarly, $B(a+1, b+1, c)=\tilde{W} \tilde{H} \tilde{W}$, with analogous expressions obtained just by shifting the parameters $a$ and $b$ by 1 . Note that $h(z)=h(z+1)$. Then (7) can be rewritten as

$$
H V=\tilde{V} \tilde{H}, \text { with } V=W C \tilde{W}^{-1}, \tilde{V}=W^{-1} C \tilde{W} .
$$

One readily finds that

$V_{j, j}=j+a, V_{j+1, j}=-j-c, \tilde{V}_{j, j}=j+b, \tilde{V}_{j+1, j}=-j-1, V_{j, k}=\tilde{V}_{j, k}=0$ otherwise.

Consequently, (7) means that

$$
h(j+k)(j+k+a)=\tilde{h}(j+k)(j+k+b+c)
$$

for all indices $j, k$. But this is actually so since

$$
\tilde{h}(z)=h(z+1)=\frac{z+a}{z+b+c} h(z),
$$

thus concluding the proof. 
Corollary 2. Let $T=T(a, b, c)$ be the three-parameter family consisting of symmetric Jacobi matrices with the entries

$T_{j, j}=j(j+c-1)+(j+a)(j+b), T_{j, j+1}=T_{j+1, j}=-\sqrt{(j+1)(j+a)(j+b)(j+c)}$,

$T_{j, k}=0$ otherwise. Then the matrices $B(a, b, c)$ and $T(a, b, c)$ commute.

Proof. Clearly, $A$ and $D^{2}$ commute and therefore, in view of (6) $), B$ and $T=C C^{T}$ commute. $T$ is a Jacobi matrix with the entries

$$
\begin{aligned}
T_{j, j} & =d(2 j-1)^{2}+d(2 j)^{2}=j(j+c-1)+(j+a)(j+b), \\
T_{j, j+1} & =T_{j+1, j}=-d(2 j) d(2 j+1)=-\sqrt{(j+1)(j+a)(j+b)(j+c)},
\end{aligned}
$$

and $T_{j, k}=0$ otherwise.

\section{Diagonalization and the spectral properties of $B(a, b, c)$}

\subsection{The associated orthogonal polynomials}

The monic orthogonal polynomials associated with a Jacobi matrix $T$ are defined by the recurrence: $P_{-1}(x)=0, P_{0}(x)=1$, and

$$
P_{j+1}(x)=\left(x-T_{j+1, j+1}\right) P_{j}(x)-\left(T_{j, j+1}\right)^{2} P_{j-1}(x) .
$$

In our case, it is convenient to modify the matrix $T$ by adding a multiple of the unit operator. The redefined diagonal entries read

$$
\begin{aligned}
T_{j, j}= & j(j+c-1)+(j+a)(j+b)-\frac{1}{4}(a+b-c)^{2} \\
= & -j(j+1)+(j-b+d)(j-c+d)+(j-a+d)(j-b+d) \\
& +(j-a+d)(j-c+d)
\end{aligned}
$$

where $d=(a+b+c) / 2$. The associated monic orthogonal polynomials, $P_{n}(x)$, coincide with the continuous dual Hahn polynomials; see Eq. 9.3.5 in [9]. We have (see also [9, Eq. 9.3.1])

$$
\begin{aligned}
P_{n}\left(x^{2}\right) & =(-1)^{n} S_{n}\left(x^{2} ; \frac{b+c-a}{2}, \frac{a+c-b}{2}, \frac{a+b-c}{2}\right) \\
& =(-1)^{n}(b)_{n}(c)_{n}{ }_{3} F_{2}\left(-n, \frac{b+c-a}{2}+i x, \frac{b+c-a}{2}-i x ; b, c ; 1\right) .
\end{aligned}
$$

The measure of orthogonality for the continuous dual Hahn polynomials is known explicitly [9, Eqs. 9.3.2, 9.3.3]. Put

$$
\rho(x)=\frac{x \sinh (2 \pi x)}{\pi^{2} \Gamma(a) \Gamma(b) \Gamma(c)}\left|\Gamma\left(\frac{b+c-a}{2}+i x\right) \Gamma\left(\frac{a+c-b}{2}+i x\right) \Gamma\left(\frac{a+b-c}{2}+i x\right)\right|^{2} .
$$


Then, for $a, b, c$ positive such that $b+c>a, a+c>b$ and $a+b \geq c$, we have the orthogonality relation

$$
\int_{0}^{\infty} P_{m}\left(x^{2}\right) P_{n}\left(x^{2}\right) \rho(x) \mathrm{d} x=(a)_{n}(b)_{n}(c)_{n} n ! \delta_{m, n} .
$$

More generally, suppose for definiteness that $0<b \leq c$ and, in agreement with (44), $0<a<b+c$. Then obviously $a+c-b>0$ but it may happen that $a+b-c<0$ (then necessarily $b<c$ ). In that case the orthogonality relation should be modified by adding a discrete part. So suppose

$$
0<b<c \text { and } 0<a<c-b
$$

(then $a<c-b<b+c)$. Put $(\lceil x\rceil$ meaning the ceiling of $x \in \mathbb{R})$

$$
N(a, b, c)=\lceil(c-a-b) / 2\rceil-1 .
$$

One has

$$
\begin{aligned}
& \int_{0}^{\infty} P_{m}\left(x^{2}\right) P_{n}\left(x^{2}\right) \rho(x) \mathrm{d} x+\frac{\Gamma(c-a) \Gamma(c-b)}{\Gamma(c) \Gamma(c-a-b)} \\
& \times \sum_{k=0}^{N(a, b, c)}\left(1+\frac{2 k}{a+b-c}\right) \frac{(-1)^{k}(a+b-c)_{k}(a)_{k}(b)_{k}}{(a-c+1)_{k}(b-c+1)_{k} k !} \\
& \quad \times P_{m}\left(-\left(\frac{a+b-c}{2}+k\right)^{2}\right) P_{n}\left(-\left(\frac{a+b-c}{2}+k\right)^{2}\right) \\
& =(a)_{n}(b)_{n}(c)_{n} n ! \delta_{m, n} .
\end{aligned}
$$

\subsection{Diagonalization of $B(a, b, c)$}

Assuming (41) let

$$
\hat{P}_{n}\left(x^{2}\right)=S_{n}\left(x^{2} ; \frac{b+c-a}{2}, \frac{a+c-b}{2}, \frac{a+b-c}{2}\right) / \sqrt{(a)_{n}(b)_{n}(c)_{n} n !} .
$$

The polynomials $\hat{P}_{n}\left(x^{2}\right)$ are normalized and $\left(\hat{P}_{0}\left(x^{2}\right), \hat{P}_{1}\left(x^{2}\right), \hat{P}_{2}\left(x^{2}\right), \ldots\right)$ is a formal eigenvector of the matrix operator $T(a, b, c)$ corresponding to the eigenvalue $x^{2}$. Let us introduce the unitary transform

$$
U: \ell^{2}\left(\mathbb{Z}_{+}\right) \rightarrow L^{2}(\mathcal{M}(a, b, c), \mathrm{d} \mu): e_{n} \rightarrow \hat{P}_{n}\left(x^{2}\right), n \in \mathbb{Z}_{+},
$$

where

$$
\mathcal{M}(a, b, c)=(0,+\infty) \cup\left\{\lambda_{k} ; k=0,1, \ldots, N(a, b, c)\right\}, \lambda_{k}=i\left(\frac{a+b-c}{2}+k\right)
$$

(the discrete part occurs if and only if $a+b-c<0), \mathrm{d} \mu(x)=\rho(x) \mathrm{d} x$ on $(0,+\infty)$ and

$$
\mu\left(\left\{\lambda_{k}\right\}\right)=\frac{(-1)^{k} \Gamma(c-a) \Gamma(c-b)}{\Gamma(c) \Gamma(c-a-b)}\left(1+\frac{2 k}{a+b-c}\right) \frac{(a+b-c)_{k}(a)_{k}(b)_{k}}{(a-c+1)_{k}(b-c+1)_{k} k !} .
$$


Remark. Note that if $0 \leq k \leq N(a, b, c)$ and (8) is true then $\mu\left(\left\{\lambda_{k}\right\}\right)>0$ as it should be. In fact, the signs of the numbers $(a+b-c)_{k},(a-c+1)_{k},(b-c+1)_{k}$ are all equal to $(-1)^{k}$. This is also a standard fact that $(\mathcal{M}(a, b, c), \mathrm{d} \mu)$ is a probability space, as seen from (10) (with $m=n=0)$.

Theorem 3. The matrix operator $B(a, b, c)$ on $\ell^{2}\left(\mathbb{Z}_{+}\right)$is unitarily equivalent to the multiplication operator by the function

$$
h(x)=\frac{1}{\Gamma(b+c-a)}\left|\Gamma\left(\frac{b+c-a}{2}+i x\right)\right|^{2}
$$

acting on $L^{2}(\mathcal{M}(a, b, c), d \mu)$.

Remark. Once more, this result should be compared to the integral operator $K_{\ell}$ which is unitarily equivalent to the multiplication operator by the function $g(\xi)$ introduced in (2) and acting on $L^{2}(\mathbb{R}, \mathrm{d} \xi)$. Again, one has to put $\ell=b+c-a-1$.

Proof. Directly from the construction it follows that $U$ diagonalizes $T$, namely $U T U^{-1}$ equals the multiplication operator by $x^{2}$. Since $B$ and $T$ commute and the spectrum of $T$ is simple, $U B U^{-1}$ is necessarily a multiplication operator, too, say by a function $h(x)$; see, for instance, Lemma 6.4 in [13] or Proposition 1.9 in Supplement 1 of [2]. One derives

$$
\begin{aligned}
h(x) & =h(x) \hat{P}_{0}\left(x^{2}\right)=U B e_{0}=\sum_{j=0}^{\infty} B_{0, j} \hat{P}_{j}\left(x^{2}\right) \\
& =\sum_{j=0}^{\infty} \frac{\Gamma(b) \Gamma(c)}{\Gamma(j+b+c) j !} S_{j}\left(x^{2} ; \frac{b+c-a}{2}, \frac{a+c-b}{2}, \frac{a+b-c}{2}\right) .
\end{aligned}
$$

More conveniently, one can rewrite the expression in terms of the Beta function,

$$
h(x)=\sum_{j=0}^{\infty} \frac{\mathrm{B}(b, j+c)}{(c)_{j} j !} S_{j}\left(x^{2} ; \frac{b+c-a}{2}, \frac{a+c-b}{2}, \frac{a+b-c}{2}\right),
$$

and so

$$
h(x)=\int_{0}^{1}(1-t)^{-1+b} t^{-1+c}\left(\sum_{j=0}^{\infty} \frac{t^{j}}{(c)_{j} j !} S_{j}\left(x^{2} ; \frac{b+c-a}{2}, \frac{a+c-b}{2}, \frac{a+b-c}{2}\right)\right) \mathrm{d} t .
$$

Making use of the generating function (see Eq. 9.3.12 in [9])

$$
\sum_{n=0}^{\infty} \frac{t^{n}}{(\alpha+\beta)_{n} n !} S_{n}\left(x^{2} ; \alpha, \beta, \gamma\right)=(1-t)_{2}^{-\gamma+i x} F_{1}(\alpha+i x, \beta+i x ; \alpha+\beta ; t)
$$

one has

$$
h(x)=\int_{0}^{1}(1-t)^{-1+(b+c-a) / 2+i x} t_{2}^{-1+c} F_{1}\left(\frac{b+c-a}{2}+i x, \frac{a+c-b}{2}+i x ; c ; t\right) \mathrm{d} t .
$$


Hence

$$
\begin{aligned}
h(x)= & \sum_{n=0}^{\infty} \frac{1}{(c)_{n} n !}\left(\frac{b+c-a}{2}+i x\right)_{n}\left(\frac{a+c-b}{2}+i x\right)_{n} \\
& \times \int_{0}^{1}(1-t)^{-1+(b+c-a) / 2+i x} t^{-1+n+c} \mathrm{~d} t \\
= & \mathrm{B}\left(\frac{b+c-a}{2}+i x, c\right) \\
& \times{ }_{2} F_{1}\left(\frac{b+c-a}{2}+i x, \frac{a+c-b}{2}+i x ; \frac{b+c-a}{2}+c+i x ; 1\right) .
\end{aligned}
$$

Recalling that [1, Eq. 15.1.20]

$$
{ }_{2} F_{1}(\alpha, \beta ; \gamma ; 1)=\frac{\Gamma(\gamma) \Gamma(\gamma-\alpha-\beta)}{\Gamma(\gamma-\alpha) \Gamma(\gamma-\beta)} \text { if } \operatorname{Re}(\gamma-\alpha-\beta)>0
$$

we finally obtain the desired expression.

\subsection{The spectrum of $B(a, b, c)$}

The function $h(x)$ defined in (14) is bounded on the positive half-line and therefore the matrix $B(a, b, c)$ represents a bounded operator on $\ell^{2}\left(\mathbb{Z}_{+}\right)$. In what follows, $B(a, b, c)$ is interpreted in this manner.

Corollary 4. The absolutely continuous part of the spectrum of $B(a, b, c)$ is simple and fills the interval $[0, M(a, b, c)]$ where

$$
M(a, b, c)=\frac{1}{\Gamma(b+c-a)} \Gamma\left(\frac{b+c-a}{2}\right)^{2} .
$$

Proof. From Theorem 3 one infers that the absolutely continuous spectrum of $B(a, b, c)$ fills the closure of $h([0,+\infty))$. We have $h(0)=M(a, b, c)$ and $h(+\infty)=0$. Moreover, for any $u$ real fixed, the function $f(x)=|\Gamma(u+i x)|^{2}$ is monotone decreasing on $(0,+\infty)$. This is immediately seen from the product formula for the Gamma function [1, Eq. 6.1.3] yielding

$$
\frac{1}{|\Gamma(u+i x)|^{2}}=\left(u^{2}+x^{2}\right) e^{2 \gamma u} \prod_{n=1}^{\infty}\left[\left(\left(1+\frac{u}{n}\right)^{2}+\left(\frac{x}{n}\right)^{2}\right) e^{-2 u / n}\right]
$$

where $\gamma$ is Euler's constant. The assertion follows.

Suppose (8) and recall the notation introduced in (9), (13)). Then the point spectrum of $T$ consists of the points $\lambda_{k}^{2}, k=0,1, \ldots, N(a, b, c)$, each eigenvalue is simple and for an eigenvector corresponding to $\lambda_{k}^{2}$ one can choose

$$
v_{k}=\sum_{j=0}^{\infty} \hat{P}_{j}\left(\lambda_{k}^{2}\right) e_{j}
$$

These vectors are also eigenvectors of $B$. 
Corollary 5. Assuming (4) and that $a+c-b, a+b-c$ are both nonnegative, the point spectrum of $B(a, b, c)$ is empty. Assuming (8), the point spectrum of $B(a, b, c)$ equals

$$
\operatorname{spec}_{p} B(a, b, c)=\left\{\beta_{0}, \beta_{1}, \ldots, \beta_{N(a, b, c)}\right\}
$$

where

$$
\beta_{k}:=h\left(\lambda_{k}\right)=\frac{\Gamma(b+k) \Gamma(c-a-k)}{\Gamma(b+c-a)},
$$

and it holds true that

$$
\beta_{0}>\beta_{1}>\ldots>\beta_{N(a, b, c)}>M(a, b, c)
$$

In particular, all eigenvalues are simple. For an eigenvector corresponding to $\beta_{k}$ one can choose the vector $v_{k}$ with the components

$$
\left\langle e_{n}, v_{k}\right\rangle=\hat{P}_{n}\left(\lambda_{k}^{2}\right)=\sqrt{\frac{(b)_{n}(c)_{n}}{(a)_{n} n !}}{ }_{3} F_{2}(-n, b+k, c-a-k ; b, c ; 1) .
$$

Its norm fulfills

$$
\left\|v_{k}\right\|^{2}=\frac{\Gamma(c) \Gamma(c-a-b-k+1) k !}{(c-a-b-2 k) \Gamma(c-a-k) \Gamma(c-b-k)(a)_{k}(b)_{k}} .
$$

Proof. Theorem 3 implies that the eigenvalues of $B(a, b, c)$ are exactly the values $h\left(\lambda_{k}\right)$. $k=0,1, \ldots, N(a, b, c)$. It remains to show (15), (16). As far as (15) is concerned, one has

$$
\Gamma(b+c-a) \beta_{k}=\Gamma\left(\frac{b+c-a}{2}+\left(\frac{a+b-c}{2}+k\right)\right) \Gamma\left(\frac{b+c-a}{2}-\left(\frac{a+b-c}{2}+k\right)\right)
$$

(recall that $a+b-c+2 k<0$ for $0 \leq k \leq N(a, b, c)$ ). Hence it suffices to observe that the function $f(t)=\log (\Gamma(t))$ is convex on the positive half-line. It is so because

$$
f^{\prime \prime}(t)=\psi^{\prime}(t)=\sum_{k=0}^{\infty} \frac{1}{(k+t)^{2}}
$$

where $\psi$ is the digamma function. It follows that, for any $u>0$, the function $g(t)=$ $\Gamma(u-t) \Gamma(u+t)$ is strictly increasing on the interval $[0, u)$.

Concerning (16) , let $f_{k} \in L^{2}(\mathcal{M}(a, b, c), \mathrm{d} \mu(x))$ be defined as follows: $f_{k}\left(\lambda_{j}\right)=\delta_{k, j}$ and $f_{k}(x) \equiv 0$ on $(0, \infty)$. Then $U v_{k}=c f_{k}$ for some $c \in \mathbb{C}$. One immediately finds that

$$
c=\left(U v_{k}\right)\left(\lambda_{k}\right)=\sum_{j=0}^{\infty} \hat{P}_{j}\left(\lambda_{k}^{2}\right)^{2}=\left\|v_{k}\right\|^{2}>0
$$

On the other hand,

$$
\left\|v_{k}\right\|^{2}=\left\|U v_{k}\right\|^{2}=c^{2}\left\|f_{k}\right\|^{2}=c^{2} \mu\left(\left\{\lambda_{k}\right\}\right)=\left\|v_{k}\right\|^{4} \mu\left(\left\{\lambda_{k}\right\}\right) .
$$


Whence

$$
\begin{aligned}
\frac{1}{\left\|v_{k}\right\|^{2}} & =\mu\left(\left\{\lambda_{k}\right\}\right) \\
& =\frac{(-1)^{k} \Gamma(c-a) \Gamma(c-b)}{\Gamma(c) \Gamma(c-a-b)}\left(1+\frac{2 k}{a+b-c}\right) \frac{(a+b-c)_{k}(a)_{k}(b)_{k}}{(a-c+1)_{k}(b-c+1)_{k} k !} \\
& =\frac{(c-a-b-2 k) \Gamma(c-a-k) \Gamma(c-b-k)(a)_{k}(b)_{k}}{\Gamma(c) \Gamma(c-a-b-k+1) k !} .
\end{aligned}
$$

This shows (16).

Remark 6. Still assuming (44), $B(a, b, c)$ is a positive bounded operator on $\ell^{2}\left(\mathbb{Z}_{+}\right)$and one has

$$
\|B(a, b, c)\|=\frac{1}{\Gamma(b+c-a)} \Gamma\left(\frac{b+c-a}{2}\right)^{2}
$$

if $a+b-c \geq 0, a+c-b \geq 0$ (at least one of the expressions is necessarily positive) and

$$
\|B(a, b, c)\|=\frac{\Gamma(b) \Gamma(c-a)}{\Gamma(b+c-a)}
$$

if $a+b-c<0, a+c-b \geq 0$, and similarly if $a+b-c \geq 0, a+c-b<0$. This means that for every square summable real sequence $\left\{\xi_{k}\right\}$,

$$
0 \leq \sum_{j=0}^{\infty} \sum_{k=0}^{\infty} B(a, b, c)_{j, k} \xi_{j} \xi_{k} \leq\|B(a, b, c)\|\left(\sum_{k=0}^{\infty} \xi_{k}^{2}\right)
$$

and the bound is best possible. Equivalently one can also say that for any real sequence $\left\{\xi_{k}\right\}$ and all $n \in \mathbb{Z}_{+}$,

$$
0 \leq \sum_{j=0}^{n} \sum_{k=0}^{n} \frac{\Gamma(j+k+a)}{\Gamma(j+k+b+c)} \xi_{j} \xi_{k} \leq\|B(a, b, c)\|\left(\sum_{k=0}^{n} \frac{\Gamma(k+a) k !}{\Gamma(k+b) \Gamma(k+c)} \xi_{k}^{2}\right),
$$

with $\|B(a, b, c)\|$ being specified in (17), (18).

The Hilbert double series inequality is a particular case for $a=\theta, b=\theta$ and $c=1$ assuming that $\theta \geq 1 / 2$. Explicitly, for any real square summable sequence $\left\{\xi_{k}\right\}$,

$$
0 \leq \sum_{j=0}^{\infty} \sum_{k=0}^{\infty} \frac{\xi_{j} \xi_{k}}{j+k+\theta} \leq \pi\left(\sum_{k=0}^{\infty} \xi_{k}^{2}\right)^{2}
$$

For $a=\theta, b=\theta, c=1$ and $0<\theta<1 / 2$ one gets the inequality

$$
0 \leq \sum_{j=0}^{\infty} \sum_{k=0}^{\infty} \frac{\xi_{j} \xi_{k}}{j+k+\theta} \leq \frac{\pi}{\sin (\pi \theta)}\left(\sum_{k=0}^{\infty} \xi_{k}^{2}\right)^{2}
$$

Again, all bounds are best possible. 


\section{Hilbert's matrix and the Bergman-Hilbert ma- trix}

\subsection{Hilbert's matrix}

As already remarked above, $H(\theta):=B(\theta, \theta, 1)$ is the generalized Hilbert matrix,

$$
H(\theta)_{j, k}=\frac{1}{j+k+\theta}, j, k=0,1,2, \ldots
$$

By our assumptions on the parameters, $\theta$ is positive. By Corollaries 4 and 5 , the absolutely continuous part of the spectrum is simple filling the interval $[0, \pi]$ independently of $\theta$. The point spectrum is nonempty if and only if $0<\theta<1 / 2$ and if so it consists of the single simple eigenvalue $\beta_{0}=\pi / \sin (\pi \theta)$.

Observing, however, that the defining expression for $H(\theta)$ is free of square roots, the range of $\theta$ can naturally be extended to $\theta \in \mathbb{R} \backslash\left(-\mathbb{Z}_{+}\right)$. The diagonalization method, as exposed in Section 3, can be applied to $H(\theta)$ without essential modifications even with this extended range. This is why we confine ourselves just to sketching some basic steps.

First of all, $H(\theta)$ commutes with the Jacobi matrix $T(\theta)$ with the entries

$T(\theta)_{j, j}=2 j(j+\theta)-1 / 4+\theta, T(\theta)_{j, j+1}=T(\theta)_{j+1, j}=-(j+1)(j+\theta), j=0,1,2, \ldots$,

and $T(\theta)_{j, k}=0$ otherwise. Referring to (11), the associated normalized orthogonal polynomials are given by

$$
\begin{aligned}
\hat{P}_{n}\left(x^{2}\right) & =\frac{1}{n !(\theta)_{n}} S_{n}\left(x^{2} ;-\frac{1}{2}+\theta, \frac{1}{2}, \frac{1}{2}\right) \\
& =\frac{(\theta)_{n}}{n !}{ }_{3} F_{2}\left(-n,-\frac{1}{2}+\theta+i x,-\frac{1}{2}+\theta-i x ; \theta, \theta ; 1\right) .
\end{aligned}
$$

It is useful to observe that the polynomials $\hat{P}_{n}\left(x^{2}\right)$ can also be expressed in terms of the Wilson polynomials [15]. By definition, for $n \in \mathbb{Z}_{+}$,

$\frac{W_{n}\left(x^{2} ; \alpha, \beta, \gamma, \delta\right)}{(\alpha+\beta)_{n}(\alpha+\gamma)_{n}(\alpha+\delta)_{n}}={ }_{4} F_{3}(-n, n+\alpha+\beta+\gamma+\delta-1, \alpha+i x, \alpha-i x ; \alpha+\beta, \alpha+\gamma, \alpha+\delta ; 1)$.

By inspection of [9, Eqs. 9.1.4, 9.1.5] one finds that

$$
\hat{P}_{n}\left(x^{2}\right)=\frac{4^{n}}{n !(\theta)_{2 n}} W_{n}\left(\frac{x^{2}}{4} ;-\frac{1}{4}+\frac{\theta}{2}, \frac{1}{4}, \frac{1}{4}+\frac{\theta}{2}, \frac{3}{4}\right) .
$$

According to (10), if $\theta<1 / 2$ then the orthogonality relation reads

$$
\int_{0}^{\infty} \hat{P}_{m}\left(x^{2}\right) \hat{P}_{n}\left(x^{2}\right) \rho(x) \mathrm{d} x+\sum_{k=0}^{N(\theta)} \mu\left(\left\{\lambda_{k}\right\}\right) \hat{P}_{m}\left(\lambda_{k}^{2}\right) \hat{P}_{n}\left(\lambda_{k}^{2}\right)=\delta_{m, n}
$$


where $N(\theta)=\lceil-1 / 2-\theta\rceil$,

$$
\rho(x)=\frac{2 x \tanh (\pi x)}{\Gamma(\theta)^{2}}\left|\Gamma\left(-\frac{1}{2}+\theta+i x\right)\right|^{2}
$$

and

$$
\lambda_{k}=i\left(-\frac{1}{2}+\theta+k\right), \mu\left(\left\{\lambda_{k}\right\}\right)=\frac{\Gamma(1-\theta)^{2}(1-2 \theta-2 k)}{k ! \Gamma(2-2 \theta-k)} .
$$

The sum on the LHS of (21) is absent if $\theta \geq 1 / 2$.

Remark 7. Strictly speaking, the orthogonality relation, as described for instance in [9, Eqs. 9.3.2, 9.3.3], covers only the cases when $\theta>0$. Nevertheless, making use of (20) and a very general complex orthogonality relation for the Wilson polynomials stated in [15], one can quite straightforwardly extend the desired formula to all values $\theta<1 / 2,-\theta \notin \mathbb{Z}_{+}$. Given $\alpha, \beta, \gamma, \delta \in \mathbb{C}$, write for short $W_{n}(z) \equiv W_{n}(z ; \alpha, \beta, \gamma, \delta)$, $n \in \mathbb{Z}_{+}$. As proved in [15],

$$
\frac{1}{2 \pi i} \int_{C} f(z) f(-z) W_{m}\left(z^{2}\right) W_{n}\left(z^{2}\right) \mathrm{d} z=\delta_{m, n} M h_{n}
$$

where

$$
\begin{aligned}
f(z) & =\frac{\Gamma(\alpha-z) \Gamma(\beta-z) \Gamma(\gamma-z) \Gamma(\delta-z)}{\Gamma(-2 z)}, \\
M & =\frac{2 \Gamma(\alpha+\beta) \Gamma(\alpha+\gamma) \Gamma(\alpha+\delta) \Gamma(\beta+\gamma) \Gamma(\beta+\delta) \Gamma(\gamma+\delta)}{\Gamma(\alpha+\beta+\gamma+\delta)},
\end{aligned}
$$

and

$$
h_{n}=\frac{n !(\alpha+\beta+\gamma+\delta-1)_{n}(\alpha+\beta)_{n}(\alpha+\gamma)_{n}(\alpha+\delta)_{n}(\beta+\gamma)_{n}(\beta+\delta)_{n}(\gamma+\delta)_{n}}{(\alpha+\beta+\gamma+\delta)_{2 n}} .
$$

The contour $C$ is the imaginary axis deformed so as to separate the set of poles of $f(z)$ from the set of poles of $f(-z)$, assuming these two sets to be disjoint. In particular, if $\alpha, \beta, \gamma$ and $\delta$ are positive, $C$ may be taken to be the imaginary axis. The orthogonality measure for the Wilson polynomials is then positive and supported on the positive real half-line. In Section 3 of [15] this result is extended to the case when $\alpha$ is negative while $\alpha+\beta, \alpha+\gamma$ and $\alpha+\delta$ are positive. The poles at $z= \pm(\alpha+k)$, with $k \in \mathbb{Z}_{+}$ and $\alpha+k<0$, then give rise to mass points of the orthogonality measure which are located on the negative real half-line. This procedure can readily be adapted to our case, with $\alpha=-1 / 4+\theta / 2, \beta=1 / 4, \gamma=1 / 4+\theta / 2$ and $\delta=3 / 4$, finally resulting in the orthogonality relation (21).

Relying on (21) one can show, similarly as in Theorem 3, that $H(\theta)$ is unitarily equivalent to the multiplication operator by the function

$$
h(x)=\frac{\pi}{\cosh (\pi x)}
$$


acting on $L^{2}(\mathcal{M}(\theta), \mathrm{d} \mu)$ where $\mathcal{M}(\theta)=(0,+\infty) \cup\left\{\lambda_{k} ; k=0,1, \ldots, N(\theta)\right\}$ and $\mathrm{d} \mu(x)=\rho(x) \mathrm{d} x$ on $(0,+\infty)$. The discrete part of $\mathcal{M}(\theta)$ occurs if and only if $\theta<1 / 2$. The corresponding unitary mapping has an analogous form as that in (12). From this explicit diagonalization one immediately deduces the full information about the spectral properties of $H(\theta)$ thus reproducing the original result due to Rosenblum as stated in [12, Theorem 5]. Rosenblum's approach was quite different than ours though an appropriate symmetry was heavily employed, too. Namely, it has been shown that $H(\theta)$ is unitarily equivalent to an integral operator on the positive half-line such that there exists an explicitly diagonalizable Sturm-Liouville operator in its commutant.

Theorem 8. For all real $\theta, \theta \neq 0,-1,-2, \ldots$, the singular continuous part of the spectrum of Hilbert's matrix $H(\theta)$ is empty and the absolutely continuous part is simple and fills the interval $[0, \pi]$. For $\theta \geq 1 / 2$, the point spectrum of $H(\theta)$ is empty. For $\theta<1 / 2$, let $N(\theta)=\lceil-1 / 2-\theta\rceil$. Then the only possible eigenvalues of $H(\theta)$ are $\pi / \sin (\pi \theta)$ and $-\pi / \sin (\pi \theta)$ whose multiplicities are respectively equal to $N(\theta) / 2+1$ and $N(\theta) / 2$ for $N(\theta)$ even, and they are both equal to $(N(\theta)+1) / 2$ for $N(\theta)$ odd.

\subsection{The Bergman-Hilbert matrix}

As another application of the general results stated in Theorem 3 and Corollaries 4 and 5 let us consider the so called Bergman-Hilbert matrix $A$ with the entries

$$
A_{j, k}=\frac{\sqrt{(j+1)(k+1)}}{(j+k+1)^{2}}, j, k \in \mathbb{Z}_{+},
$$

which has been introduced and studied as an operator on $\ell^{2}\left(\mathbb{Z}_{+}\right)$in [4, 3]. It is shown in [3, Prop. 2] that the essential spectrum of $A$ equals the interval $[0,1]$. We can make this analysis more complete by identifying the absolutely continuous spectrum of $A$.

Proposition 9. The absolutely continuous spectrum of the Bergman-Hilbert matrix $A$, regarded as an operator on $\ell^{2}\left(\mathbb{Z}_{+}\right)$, is simple and fills the interval $[0,1]$.

Proof. Referring to (3), let $B=B(1,1,2)$. Then

$$
B_{j, k}=\frac{\sqrt{(j+1)(k+1)}}{(j+k+1)(j+k+2)}, j, k \in \mathbb{Z}_{+},
$$

From Corollary 4 we know that the absolutely continuous spectrum of $B$ is simple filling the interval $[0,1]$.

Let us show that $Z:=A-B$ is a trace class operator. We have

$$
Z_{j, k}=\frac{\sqrt{(j+1)(k+1)}}{(j+k+1)^{2}(j+k+2)} .
$$

Expanding

$$
\frac{1}{(j+k+1)^{2}}=\sum_{s=0}^{\infty} \frac{(s+1) j^{s} k^{s}}{(j+1)^{s+2}(k+1)^{s+2}}
$$


we can write

$$
Z=\sum_{s=0}^{\infty} T_{s}, \quad \text { where } T_{s}=(s+1) J_{s} H(1) J_{s}, J_{s}=\operatorname{diag}\left\{\frac{j^{s}}{(j+1)^{s+3 / 2}} ; j \in \mathbb{Z}_{+}\right\},
$$

where we have used the notation (19). From Theorem 8 we know that $H(1)$ is positive and so is $T_{s}$. Consequently $\left(\|\cdot\|_{1}\right.$ standing for the trace norm),

$$
\sum_{s=0}^{\infty}\left\|T_{s}\right\|_{1}=\sum_{s=0}^{\infty} \sum_{j=0}^{\infty}\left(T_{s}\right)_{j, j}=\frac{1}{2} \sum_{j=0}^{\infty} \sum_{s=0}^{\infty} \frac{(s+1) j^{2 s}}{(j+1)^{2 s+4}}=\frac{\pi^{2}}{16} .
$$

The space of trace class operators is complete and therefore $Z$ is, too, a trace class operator.

To conclude the proof we recall that the absolutely continuous spectrum is known to be invariant with respect to trace class perturbations.

\section{Acknowledgments}

One of the authors (P.Š.) wishes to acknowledge gratefully partial support from grant No. GA13-11058S of the Czech Science Foundation.

\section{References}

[1] M. Abramowitz, I. A. Stegun: Handbook of Mathematical Functions, (Dover Publications, New York, 1972).

[2] F. A. Berezin, M. A. Shubin: The Schrödinger Equation, (Kluwer Academic Publishers, Dordrecht, 1991).

[3] C. Davis, P. Ghatage: On the spectrum of the Bergman-Hilbert matrix II, Canad. Math. Bull. 33 (1990) 60-64.

[4] P. G. Ghatage: On the spectrum of the Bergman-Hilbert matrix, Linear Algebra Appl. 97 (1987) 57-63.

[5] F. A. Grünbaum: Toeplitz matrices commuting with tridiagonal matrices, Linear Alg. Appl. 40 (1981) 25-36.

[6] F. A. Grünbaum: A remark on Hilbert's matrix, Linear Alg. Appl. 43 (1982) 119-124.

[7] T. Kato: On positive eigenvectors of positive infinite matrices, Commun. Pure Appl. Math. 11 (1958) 573-586.

[8] G. H. Hardy, J. E. Littlewood, G. Pólya: Inequalities, (Cambridge University Press, London, 1934). 
[9] R. Koekoek, P. A. Lesky, R. F. Swarttouw: Hypergeometric Orthogonal Polynomials and Their q-Analogues, (Springer-Verlag, Berlin, 2010).

[10] P. Otte: Diagonalization of the Hilbert matrix, ICDESFA 2005 conference, Munich (Germany), 25-30 July 2005; URL:

http://homepage.ruhr-uni-bochum.de/Peter.Otte/publications.html.

[11] V. V. Peller: Hankel Operators and Their Applications, (Springer-Verlag, NewYork, 2003).

[12] M. Rosenblum: On the Hilbert Matrix, II, Proc. Amer. Math. Soc. 9 (1958) 581585.

[13] V. S. Varadarajan: Geometry of Quantum Theory, 2nd ed., (Springer, Berlin, 1985).

[14] H. S. Wilf: Finite Sections of Some Classical Inequalities, (Springer-Verlag, New York, 1970).

[15] J. A. Wilson: Some hypergeometric orthogonal polynomials, SIAM J. Math. Anal. 11 (1980) 690-701. 